\title{
FIXED POINT SETS OF METRIC AND NONMETRIC SPACES
}

\author{
BY
}

JOHN R. MARTIN AND WILLIAM WEISS ${ }^{1}$

\begin{abstract}
A space $X$ is said to have the complete invariance property (CIP) if every nonempty closed subset of $X$ is the fixed point set of some self-mapping of $X$. It is shown that connected subgroups of the plane and compact groups need not have CIP, and CIP need not be preserved by self-products of Peano continua, nonmetric manifolds or 0-dimensional spaces. Sufficient conditions are given for an infinite product of spaces to have CIP. In particular, an uncountable product of real lines, circles or two-point spaces has CIP. Examples are given which contrast the behavior of CIP in the nonmetric and metric cases, and examples of spaces are given where the existence of CIP is neither provable nor refutable with the usual axioms of set theory.
\end{abstract}

1. Introduction. All spaces considered in this paper will be regular Hausdorff spaces. A subset $K$ of a space $X$ is called a fixed point set of $X$ if there is a mapping (continuous function) $f: X \rightarrow X$ such that $f(x)=x$ iff $x \in K$. The singleton subsets and $X$ itself are always fixed point sets of $X$, and an example of H. Cook [2, p. 245] shows that there exists a nondegenerate continuum where these are the only fixed point sets. A space which has the property that each of its nonempty closed subsets is a fixed point set is said (see [14, p. 553]) to have the complete invariance property (CIP). Some classes of spaces known to have CIP are the class of spaces which admit a strongly convex metrization [14, p. 554], compact finite-dimensional (metrizable) manifolds [12, p. 420], 1-dimensional Peano continua [10], locally compact metric groups or any metric group which contains an arc [9, p. 1028], and locally finite simplicial complexes with the weak topology [5, p. 171]. A survey and additional references concerning CIP may be found in [12].

In this paper we give conditions which ensure that certain product spaces and topological groups have CIP and give examples of products and groups failing to have CIP. In particular, we answer some questions posed by $\mathrm{H}$. Schirmer (see Questions 3 and 6 in [12]) by showing that CIP need not be preserved by self-products and that neither connected metric groups nor compact groups need have CIP. Various examples are given which illustrate the pathology of CIP with

Received by the editors July 28, 1983. Presented to the Society, August 26, 1982 (Toronto Meeting).

1980 Mathematics Subject Classification. Primary 54H25, 54C99.

Key words and phrases. Fixed point set, complete invariance property, (Cartesian) product space, topological group, continuum hypothesis, Martin's Axiom.

${ }^{1}$ The first author was partially supported by NSERC Grant A8205, and the second author partially supported by NSERC Grant A3185. 
respect to nonmetrizable products. For instance, it is shown that the long line has CIP while its square does not, and therefore CIP need not be preserved by self-products of nonmetric manifolds. Also, it is shown that the self-product $K^{\lambda}$ of $\lambda$ copies of the Sorgenfrey line $K$ has CIP if $\lambda=1$ or $\lambda \geqslant \mathbf{c}$ (the cardinal of the continuum), but not if $1<\lambda \leqslant \boldsymbol{\aleph}_{0}$. In the case of $\beta N$, the Stone-Čech compactification of the integers, it is shown that the self-product $(\beta N)^{\mathbf{c}}$ has CIP while $\beta N$ does not. Finally, examples of spaces are given where the existence of CIP is neither provable nor refutable with the usual axioms of set theory. Furthermore, it is shown that additional axioms can be chosen so as to imply the existence (or nonexistence) of CIP in these spaces.

2. Metrizable products and groups. We follow the definitions found in [11, p. 134] and say that a space $X$ has property $W$ if for every point $p \in X$ there is a deformation $H: X \times I \rightarrow X$ such that $H(x, t) \neq x$ if $x \neq p$ and $t>0$. If $X$ satisfies the stronger condition that $H(x, t) \neq x$ whenever $t>0$, we say that $X$ has property $W$ (strong). It is readily seen that if $X$ has property $W$ (strong) and $Y$ is an arbitrary space, then the (Cartesian) product space $X \times Y$ has property $W$ (strong). We now show that a slight modification of a proof of L. E. Ward, Jr. [14, p. 554] concerning metric spaces yields the same result for perfectly normal spaces. Recall that a $T_{1}$-space $X$ is perfectly normal iff every closed subset $K$ of $X$ is such that $K=f^{-1}(0)$ for some mapping $f: X \rightarrow I[4,1.5 .19$, p. 69].

\subsection{WARD's LEMMA. Any perfectly normal space X having property $W$ has CIP.}

Proof. Let $K$ be a nonempty closed subset of $X$ and suppose $p \in K$. Then there is a deformation $H: X \times I \rightarrow X$ such that $H(x, t) \neq x$ if $x \neq p$ and $t>0$. Since $X$ is perfectly normal there is a mapping $g: X \rightarrow I$ such that $g(x)=0$ iff $x \in K$. Define a mapping $f: X \rightarrow X$ by $f(x)=H(x, g(x))$ if $x \in X$. Then $K$ is the fixed point set of $f$ and $X$ has CIP as required.

In $[9$, p. 1028] it is shown that any metric group which is either locally compact or contains an arc has CIP. The question as to whether every metric group has CIP has been raised in $[9$, p. 1038 and 12, p. 422]. The following result shows that a metric group need not have CIP if it fails to satisfy the condition that either it is locally compact or contains an arc. In fact, it is shown that even a connected subgroup of the Euclidean plane $R^{2}$ need not have CIP.

2.2. TheOREM. There is a connected subgroup of the plane $R^{2}$ which does not have CIP.

Proof. In [6, p. 118] F. B. Jones shows that there is a (totally discontinuous) real-valued function $f: R \rightarrow R$ satisfying the functional equation $f(x)+f(y)=$ $f(x+y)$ such that $X=\{(x, f(x)) \mid x \in R\}$ is a dense and connected subset of $R^{2}$ with the property that $X \cap P \neq \varnothing$ if $P$ is any perfect subset of $R^{2}$ which is not contained in a countable union of vertical lines. Thus $X$ is a connected subgroup of $R^{2}$, and we claim that the subset $K=\{(x, f(x)) \mid f(x) \leqslant 0$ or $f(x) \geqslant 1\}$ is not a fixed point set of $X$. 
Suppose $h: X \rightarrow X$ is a mapping whose fixed point set is $K$. Then there is an extension of $h$ to a mapping $H: Y \rightarrow R^{2}$ where $Y$ is a $G_{\delta}$-set in $R^{2}$ which contains $X$ $\left[4,4.3 .20\right.$, p. 341]. Hence we can write $R^{2}-Y=\bigcup_{i=1}^{\infty} F_{i}$ where each $F_{i}$ is a closed subset of $R^{2}$. We now show that $R^{2}-Y$ is contained in a countable union of vertical lines. To see this, suppose the contrary is true. Then some $F_{n}$ is not contained in a countable union of vertical lines and, by the Cantor-Bendixson theorem $[4, \mathrm{p}$. 85], we can write $F_{n}=P \cup C$ where $P$ is a perfect set and $C$ is countable. It follows that $X \cap P \neq \varnothing$ which is a contradiction.

Let $[p, q]$ denote the interval in $X$ defined by $[p, q]=\{(x, f(x)) \mid p \leqslant x \leqslant q\}$. Then $[p, q]$ is connected and irreducible between its endpoints $[6$, p. 119]. Thus, if $[a, b]$ is an arbitrary interval whose endpoints lie on the $x$-axis, it follows that $h([a, b])$ is a connected set containing $[a, b]$. We claim that $h([a, b])=[a, b]$. To see this, suppose $a<c<b$ and $h(c, f(c))=(d, f(d)) \notin[a, b]$. Then $0<f(c)<1$ and without loss of generality we may assume that $(c, f(c))$ belongs to a vertical line contained in $Y$. Let $M$ denote the vertical line segment joining $(c, 0)$ to $(c, 1)$ and let $V$ be a bounded neighborhood of $H(M)$ in $R^{2}$. Then there is a neighborhood $U$ of $M$ in $Y$ such that $H(U) \subset V$. Let $[s, t]$ be a subinterval of $[a, b]$ containing $(c, f(c))$ whose endpoints lie on the $x$-axis and is such that $B=[s, t] \cap(X-K) \subset U$. Since $h$ is the identity on $K$ and $h([s, t])$ is a connected set containing $(d, f(d))$, it follows that $h(B)$ is not bounded. This contradiction shows that $h([a, b])=[a, b]$. Consequently, the fixed point set of $h$ must be all of $X$ and this contradiction completes the proof.

2.3. COROLlaRY. There is a connected metric group having CIP which contains a subgroup, failing to have CIP, as a strong deformation retract.

Proof. Let $X$ be the connected metric group defined in 2.2 (i.e., $X$ is the Jones group). Then $R \times X$ has CIP by Ward's Lemma (or [9, p. 1028]) and contains $\{0\} \times X$ as a strong deformation retract.

The question as to whether CIP is preserved by self-products has been asked in $[12$, p. 426]. In the following result we show that a modification of the ideas found in Examples 3.5 of [11, p. 135] can be used to prove that CIP need not be preserved by self-products of Peano continua.

\subsection{TheOREM. There is a 1-dimensional planar Peano continuum $X$ such that $X \times X$} does not have CIP.

Proof. Let $Z_{n}$ denote the circle in the closed upper half-plane $R_{+}^{2}$ with center $(0,1 / n)$ and radius $1 / n$ for $n=1,2, \ldots$. The space $Z=\bigcup_{n=1}^{\infty} Z_{n}$ is called a Hawaiian earring and $(0,0)$ is called its point of tangency. For $i=1,2, \ldots$, let $B_{i}=\bigcup_{n=1}^{\infty} Z_{n}^{i}$ form a disjoint null sequence of homeomorphs of $Z$ lying in $R_{+}^{2}$ such that the point of tangency of $B_{i}$ is $p_{i}=(1 /(i+1), 0)$. Define

$$
X=A \cup \bigcup_{i=1}^{\infty} B_{i}
$$


where $A$ denotes the closed unit interval on the $x$-axis joining the origin $a_{0}=(0,0)$ to the point $a_{1}=(1,0)$. Then $X$ is a 1-dimensional planar Peano continuum and hence has CIP by [10, p. 147]. Let $C, D$ denote the closed subsets of $X \times X$ defined by

$$
\begin{aligned}
& C=\left\{\left(a_{0}, a_{0}\right)\right\} \cup \bigcup_{k=1}^{\infty} B_{2 k-1} \times\left\{a_{0}\right\}, \\
& D=\left\{\left(a_{0}, a_{1}\right)\right\} \cup \bigcup_{k=1}^{\infty} B_{2 k} \times\left\{a_{1}\right\} .
\end{aligned}
$$

We show that $C \cup D$ cannot be a fixed point set of $X \times X$.

First we remark that elementary homotopy arguments can be used to show that the 1-sphere $Z_{n}^{i} \times\left\{p_{k}\right\}$ cannot be deformed in the torus $Z_{n}^{i} \times Z_{m}^{k}$ into the 1-sphere $\left\{p_{i}\right\} \times Z_{m}^{k}$ and, consequently, cannot be deformed in $Z_{n}^{i} \times \bigcup_{m=1}^{\infty} Z_{m}^{k}$ into $\left\{p_{i}\right\} \times$ $\bigcup_{m=1}^{\infty} Z_{m}^{k}$. Using this fact and the construction of $X \times X$, it is possible to show that no 1-sphere of the form $Z_{n}^{i} \times\left\{a_{j}\right\}, j=0,1$, can be deformed to a point in $X \times X$.

Now we suppose there is a mapping $f: X \times X \rightarrow X \times X$ whose fixed point set is $C \cup D$ and then obtain a contradiction. In what follows, in order to facilitate notation, we identify $A=I \times\{0\}$ with $I$ and further identify $Z_{n}^{i}$ with $Z_{n}^{i} \times\{0\}$.

Claim 1. If $q \in\left\{p_{i}\right\} \times I$, then $X \times X$ is not locally contractible at $f(q)$.

Proof. We prove the claim for the case when $i$ is odd. The case when $i$ is even is similar.

Suppose $X \times X$ is locally contractible at $f(q)$. Let $U$ be a neighborhood of $f(q)$ which is contractible in $X \times X$ and let $\beta: U \times I \rightarrow X \times X$ be a deformation which deforms $U$ to a point in $X \times X$. Since $f$ is continuous at $q$, there is a neighborhood $V$ of $q$ in $X \times X$ such that $f(V) \subset U$. By construction there is an integer $N$ such that $Z_{N}^{i} \times\{b\} \subset V$ where $q=\left(p_{i}, b\right)$. Let $\alpha: Z_{N}^{i} \times I \rightarrow Z_{N}^{i} \times[0, b]$ be a deformation which deforms $Z_{N}^{i}=Z_{N}^{i} \times\{0\}$ onto $Z_{N}^{i} \times\{b\}$. Define a homotopy $H: Z_{N}^{i} \times I \rightarrow X$ $\times X$ by

$$
H(x, t)= \begin{cases}f \alpha(x, 2 t) & \text { if } 0 \leqslant t \leqslant 1 / 2 \\ \beta(f \alpha(x, 1), 2 t-1) & \text { if } 1 / 2 \leqslant t \leqslant 1\end{cases}
$$

It is easy to check that $H$ is a deformation which deforms $Z_{N}^{i}=Z_{N}^{i} \times\{0\}$ to a point in $X \times X$. This contradiction completes the proof of Claim 1 .

Claim 2. If $q \in\left\{p_{i}\right\} \times I$, then $f(q) \in\left\{p_{i}\right\} \times I$.

Proof. From the remarks preceding Claim 1 it follows that $Z_{n}^{i} \times\{0\}$ cannot be deformed in $B_{i} \times I \cup I \times B_{k}$ into $I \times B_{k}$. It then follows that no 1-sphere of the form $Z_{n}^{i} \times\{0\}$ can be deformed in $X \times X$ into a set of the form $I \times B_{k}$.

As in Claim 1 we prove the case when $i$ is odd and omit the analogous case when $i$ is even. Thus suppose $i$ is odd, $q=\left(p_{i}, b\right)$ and $f(q) \notin\left\{p_{i}\right\} \times I$. From Claim 1 it follows that

$$
f\left(\left\{p_{i}\right\} \times[0, b]\right) \subset \bigcup_{j=1}^{\infty}\left\{p_{j}\right\} \times I \cup \bigcup_{j=1}^{\infty} I \times\left\{p_{j}\right\}
$$


Without loss of generality we may assume that, for some $k, f(q) \in J \times\left\{p_{k}\right\}$ where $J$ is a closed subinterval of $I$ which contains no $p_{j}, j=1,2, \ldots$. Let $U$ be a neighborhood of $f(q)$ which is disjoint from $\left\{p_{i}\right\} \times I$ and which contains $J \times B_{k}$ as a deformation retract. Using techniques found in the proof of Claim 1, we find a 1-sphere $Z_{M}^{i}=Z_{M}^{i} \times\{0\}$ and a deformation $H: Z_{M}^{i} \times I \rightarrow X \times X$ such that $H\left(Z_{M}^{i} \times\{1\}\right) \subset U$. Since $J \times B_{k}$ is a deformation retract of $U$ it follows that there is a deformation of $Z_{M}^{i}=Z_{M}^{i} \times\{0\}$ in $X \times X$ which deforms $Z_{M}^{i}$ into $J \times B_{k}$. This contradiction completes the proof of Claim 2.

Now $f\left(\left\{p_{i}\right\} \times I\right) \subset\left\{p_{i}\right\} \times I$ for $i=1,2, \ldots$, and

$$
\left\{p_{i}\right\} \times I \cap(C \cup D)= \begin{cases}\left\{\left(p_{i}, 0\right)\right\} & \text { if } i \text { is odd } \\ \left\{\left(p_{i}, 1\right)\right\} & \text { if } i \text { is even }\end{cases}
$$

Thus, if $f_{2}$ is defined by $f\left(p_{i}, t\right)=\left(p_{i}, f_{2}\left(p_{i}, t\right)\right)$, it follows that for $0<t<1$, $f_{2}\left(p_{i}, t\right)<t$ if $i$ is odd and $f_{2}\left(p_{i}, t\right)>t$ if $i$ is even. Therefore $f\left(a_{0}, t\right)=\left(a_{0}, t\right)$ for all $t \in I$. Hence $\left\{a_{0}\right\} \times I=\left\{a_{0}\right\} \times A$ is a subset of the fixed point set of $f$ and this contradiction completes the proof.

2.5. REMARK. We remark that by replacing the 1 -spheres with higher-dimensional spheres in the proof of Theorem 2.4, it is possible to obtain examples which show that CIP need not be preserved by self-products of continua which are locally connected in higher dimensions. Since this involves only the proof of Theorem 2.4 together with some ideas which can be found in Examples 3.6 of [11, p. 136], we omit the details.

3. The nonmetric case. The prototypes for metric spaces having CIP are the closed unit interval $I$, the circle $S^{1}$, or the product of any metric space with $S^{1}$. In [14, p. 555] L. E. Ward, Jr. shows that the nonmetric case is substantially different by giving an example of a nonmetric order arc which fails to have CIP. To begin with, we further illustrate this difference by showing that the long line $L$ (see [4, p. 297]) and $L \times S^{1}$ have CIP while their Stone-Cech compactifications $L^{*}$ and $L^{*} \times S^{1}$ do not. In particular, the latter shows that the perfect normality condition in Ward's Lemma is essential.

3.1. ExAmple. Let $K$ be a nonempty closed subset of $L$ whose greatest lower bound is denoted by $a$. If $K$ has a least upper bound, say $b$, then the closed interval $[a, b]$ contains $K$ and is homeomorphic to $I$. Since $[a, b]$ is a retract of $L$ having CIP, it follows that $K$ is a fixed point set of $L$. If $K$ is not bounded above, then $L-K$ is the union of a disjoint family of open intervals, say $\left\{\left(a_{\alpha}, b_{\alpha}\right) \mid \alpha \in \Lambda\right\}$. For each $\alpha \in \Lambda$, let $f_{\alpha}$ denote an autohomeomorphism of the closed interval $\left[a_{\alpha}, b_{\alpha}\right]$ whose fixed point set is $\left\{a_{\alpha}, b_{\alpha}\right\}$. Define a mapping $f: L \rightarrow L$ by

$$
f(x)= \begin{cases}a & \text { if } x \leqslant a, \\ x & \text { if } x \in K, \\ f_{\alpha}(x) & \text { if } x \in\left(a_{\alpha}, b_{\alpha}\right), \alpha \in \Lambda .\end{cases}
$$

Then $K$ is the fixed point set of $f$ and $L$ has CIP.

3.2. EXAMPLE. Let $\omega_{1}$ denote the first uncountable ordinal and let $L^{*}=L \cup\left\{\omega_{1}\right\}$ denote the long segment (see [4, p. 297]). We claim that the set $K=\left\{0, \omega_{1}\right\}$ of 
endpoints of the ordered continuum $L^{*}$ is not a fixed point set of $L^{*}$. To see this, suppose $f$ is a self-mapping of $L^{*}$ whose fixed point set is $K$. Then either (i) $x<f(x)$ for all $x \in L^{*}-K$, or (ii) $x>f(x)$ for all $x \in L^{*}-K$. Suppose we have (i) and $p \in L^{*}-K$. Then the positive semi-orbit $p, f(p), f^{2}(p), \ldots$ has a limit $q \in L^{*}-K$ and therefore $f(q)=q$. Hence we suppose we have (ii) and $x_{1} \in L^{*}-K$. Then there is a sequence of points $x_{1}, x_{2}, x_{3}, \ldots$ in $L^{*}-K$ such that $f\left(x_{n+1}\right)=x_{n}$ for $n=1,2, \ldots$. It follows that $\lim _{n \rightarrow \infty} x_{n}=y$ for some point $y \in L^{*}-K$ and $f(y)=y$. This contradiction shows that $K$ is not a fixed point set of $L^{*}$.

3.3. REMARKs. The two preceding examples show that the order $\operatorname{arc} L^{*}=\left[0, \omega_{1}\right]$ does not have CIP even though $L=L^{*}-\left\{\omega_{1}\right\}$ is a nonmetric manifold having CIP. We remark that order arcs constructed by the same method used to construct $L^{*}$ can be used to obtain a variety of nonmetric simple closed curves which may or may not have CIP. For instance, a nonmetric simple closed curve $C$ failing to have CIP may be obtained by identifying the endpoints of the order arc $\left[0,2 \omega_{1}\right]$ and checking that $\left\{0, \omega_{1}\right\}$ is not a fixed point set of $C$. On the other hand, it is easy to check that the nonmetric simple closed curve obtained by identifying the endpoints of $\left[0, \omega_{1}\right]$ does have CIP.

3.4. ExAmple. Let $q \in S^{1}$ and $h: L^{*} \rightarrow L^{*} \times S^{1}$ be an embedding defined by $h(x)=(x, q)$ for each $x$ in $L^{*}=\left[0, \omega_{1}\right]$. Suppose $f$ is a self-mapping of $L^{*} \times S^{1}$ whose fixed point set is $\left\{h(0), h\left(\omega_{1}\right)\right\}$. Let $V_{1}, V_{2}, \ldots$ be a decreasing sequence of open intervals in $S^{1}$ such that $\bigcap_{i=1}^{\infty} V_{i}=\{q\}$ and let $\pi: L^{*} \times S^{1} \rightarrow S^{1}$ denote the natural projection mapping. Consider the mapping $\pi f h: L^{*} \rightarrow S^{1}$. Then

$$
(\pi f h)^{-1}(q)=(\pi f h)^{-1}\left(\bigcap_{i=1}^{\infty} V_{i}\right)=\bigcap_{i=1}^{\infty}(\pi f h)^{-1}\left(V_{i}\right)
$$

is a countable intersection of open sets in $L^{*}$ containing $\omega_{1}$. Since $\left\{\omega_{1}\right\}$ is not a $G_{\delta}$-set in $L^{*}$, there is a point $\alpha \in L$ such that $\left[\alpha, \omega_{1}\right] \subset(\pi f h)^{-1}\{q\}$. Therefore $f h\left(\left[\alpha, \omega_{1}\right]\right) \subset \pi^{-1}(q)=h\left(L^{*}\right)$. Arguments similar to those used in the proof that $L^{*}$ does not have CIP can be used to show that there is a point $p \in\left(\alpha, \omega_{1}\right)$ such that $f(h(p))=h(p)$. This contradiction shows that $L^{*} \times S^{1}$ does not have CIP.

3.5. EXAMPLE. Let $K$ be a nonempty closed subset of $L \times S^{1}$. If $K$ is compact, then $K$ is a subset of a retract of $L \times S^{1}$ which is homeomorphic to $I \times S^{1}$. Since $I \times S^{1}$ has CIP by Ward's Lemma, it follows that $K$ is a fixed point set of $L \times S^{1}$. Hence we assume that $K$ is not compact and let

$$
Y=\left\{y \in S^{1} \mid\{x \mid(x, y) \in K\} \text { is unbounded in } L\right\} .
$$

In order to show that $K$ is a fixed point set of $L \times S^{1}$, we first prove two claims.

Claim 1. There exists an $\alpha_{0} \in \omega_{1}$ such that if $x>\alpha_{0}$ and $(x, y) \in K$, then $y \in Y$.

Proof. Suppose to the contrary that, for every $\alpha \in \omega_{1}$, there exist elements $x_{\alpha}>\alpha$ and $y_{\alpha} \notin Y$ such that $\left(x_{\alpha}, y_{\alpha}\right) \in K$. Since each $y_{\alpha} \notin K$, the set $\left\{y_{\alpha} \mid \alpha \in \omega_{1}\right\}$ must be uncountable. Let $q$ be an element of this set which is a complete accumulation point of the set. We use $q$ to obtain a contradiction.

Let $\beta_{0} \in \omega_{1}$. For each $n \in \omega_{0}$, pick $\beta_{n+1}>\beta_{n}$ such that:

(i) $x_{\beta_{n+1}}>x_{\beta_{n}}$, and 
(ii) $\left\|q-y_{\beta_{n+1}}\right\|<1 /(n+1)$ where $\|\cdot\|$ denotes the usual norm on $R^{2}$.

Let $x=\sup \left\{x_{\beta_{n}} \mid n \in \omega_{0}\right\}$. Thus $(x, q) \in K$ since $K$ is closed. Since $\beta_{0}$ was arbitrary and $x>\beta_{0}$, we conclude that $q \in Y$ which is a contradiction.

Claim 2. There is a closed unbounded set $C \subset \omega_{1}-\alpha_{0}$ such that for every $\alpha_{1}, \alpha_{2} \in C$,

$$
\left\{y \mid\left(\alpha_{1}, y\right) \in K\right\}=\left\{y \mid\left(\alpha_{2}, y\right) \in K\right\} .
$$

Proof. Let $D$ be a countable dense subset of $Y$. For each $y \in D$, the set $C_{y}=\left\{\alpha \in \omega_{1} \mid(\alpha, y) \in K\right\}$ is a closed unbounded subset of $\omega_{1}$. Hence $C=\left(\omega_{1}-\right.$ $\left.\alpha_{0}\right) \cap \cap\left\{C_{y} \mid y \in D\right\}$ is also closed and unbounded. We show that $C$ is as required by showing that, for each $\alpha \in C,\{y \mid(\alpha, y) \in K\}=Y$.

Now for any $\alpha \in C$ and any $y \in D,(\alpha, y) \in K$, so $D \subset\{y \mid(\alpha, y) \in K\}$. Thus we need only show that if $\alpha \in C$ and $(\alpha, y) \in K$, then $y \in Y$. But the latter follows from Claim 1.

Now pick a subset $\left\{c_{\alpha} \mid \alpha \in \omega_{1}\right\}$ of $C$ which is isomophic to $\omega_{1}$. For each $\alpha \in \omega_{1}$, the subinterval of $L$ defined by $L_{\alpha}=\left[c_{\alpha}, c_{\alpha+1}\right]$ is homeomorphic to the closed unit interval $I$ under an order-preserving homeomorphism $h_{\alpha}$. Let $\rho_{\alpha}$ denote the metric on $L_{\alpha}$ defined by $\rho_{\alpha}(x, y)=\left\|h_{\alpha}(x)-h_{\alpha}(y)\right\|$ for all $x, y \in L_{\alpha}$. For each $\alpha \in \omega_{1}$, let $H_{\alpha}: L_{\alpha} \times I \rightarrow L_{\alpha}$ be the deformation defined by

$$
H_{\alpha}(x, t)=h_{\alpha}^{-1}\left((1-t) h_{\alpha}(x)+t\left(h_{\alpha}(x)\right)^{2}\right) \quad \text { if }(x, t) \in L_{\alpha} \times I .
$$

We note that $H_{\alpha}$ is a deformation such that, for each $t>0$, the fixed point set of $H_{\alpha}(\cdot, t)$ is $\left\{c_{\alpha}, c_{\alpha+1}\right\}$.

Set $K_{0}=K \cap\left(\left[0, c_{0}\right] \times S^{1}\right)$, and let $\rho$ be a metric on $S^{1}$ which is bounded by 1 . Let $G: S^{1} \times I \rightarrow S^{1}$ be the deformation defined by

$$
G\left(e^{i \theta}, t\right)=e^{i(\theta+t)} \quad \text { if }\left(e^{i \theta}, t\right) \in S^{1} \times I .
$$

Define a function $f: L \times S^{1} \rightarrow L \times S^{1}$ by

$$
f(x, y)=\left\{\begin{aligned}
\left(x, G\left(y, \rho\left((x, y), K_{0}\right)\right)\right) & \text { if }(x, y) \in\left[0, c_{0}\right] \times S^{1}, \\
\left(H_{\alpha}\left(x, \rho_{\alpha}((x, y), K)\right), G\left(y, \rho\left(\left(c_{0}, y\right), K_{0}\right)\right)\right) & \text { if }(x, y) \in L_{\alpha} \times S^{1}, \alpha \in \omega_{1} .
\end{aligned}\right.
$$

From the construction it follows that $f$ is continuous and the fixed point set of $f$ is $K$ as required.

From the preceding examples one might be tempted to conjecture that nonmetric manifolds have CIP. However, the following result shows that even the self-product of a 1-dimensional nonmetric manifold having CIP need not have CIP.

3.6. THEOREM. The self-product $L \times L$ of the long line $L$ does not have CIP.

Proof. Let $K=L \times\{0\} \cup\{0\} \times L$. We suppose that $K$ is the fixed point set of a mapping $f: L \times L \rightarrow L \times L$ and establish a sequence of claims to obtain a contradiction.

Let $f_{1}, f_{2}$ be defined by $f(x, y)=\left(f_{1}(x, y), f_{2}(x, y)\right)$, and let $\Delta$ denote the diagonal set $\{(x, x) \mid x \in L\}$. 
Claim 1. At least one of $f_{1}, f_{2}$ is bounded on $\Delta$.

PROOF. We suppose otherwise and show that $f$ has a fixed point not in $K$. Since each $f_{i}$ is unbounded on $\Delta$, it is possible to obtain a (strictly) monotonically increasing sequence $\left\{x_{n}\right\}$ such that:

(i) $f_{2}\left(x_{2 n+2}, x_{2 n+2}\right)>x_{2 n+1}>f_{2}\left(x_{2 n}, x_{2 n}\right)$ and

(ii) $x_{2 n+2}>f_{1}\left(x_{2 n+1}, x_{2 n+1}\right)>x_{2 n}$.

Let $p=\lim _{n \rightarrow \infty} x_{n}$. By construction we have $p=\lim _{n \rightarrow \infty} f_{1}\left(x_{2 n+1}, x_{2 n+1}\right)$ and $p=\lim _{n \rightarrow \infty} f_{2}\left(x_{2 n+2}, x_{2 n+2}\right)$. Hence by the continuity of $f$ we have $f(p, p)=$ $(p, p) \notin K$.

Now, without loss of generality, we assume that $f_{1}$ is bounded on $\Delta$ by $\beta \in \omega_{1}$.

For each limit ordinal $\alpha \in \omega_{1}$ we pick an ordinal $\phi(\alpha)<\alpha$ such that $f_{1}(\alpha, \phi(\alpha))$ $<\beta+1$. By the Pressing Down Lemma [3, 3.4, p. 131] there is an uncountable set $A \subset \omega_{1}$ and an ordinal $\gamma \in \omega_{1}$ such that, for every $\alpha \in A, \phi(\alpha)=\gamma$. Thus $f_{1}(\alpha, \gamma)<$ $\beta+1$ for all $\alpha \in A$.

Claim 2. The set $\left\{x \in L \mid f_{1}(x, \gamma)>\beta+2\right\}$ is bounded.

Proof. Suppose the contrary is true. Then we obtain sequences $\left\{x_{n}\right\}$ and $\left\{\alpha_{n}\right\}$ such that

$$
x_{0}<\alpha_{0}<x_{1}<\alpha_{1}<x_{2}<\cdots<\alpha_{n}<x_{n+1}<\alpha_{n+1}<\cdots
$$

and, for each $n$,

$$
f_{1}\left(x_{n}, \gamma\right)>\beta+2 \text { and } f_{1}\left(\alpha_{n}, \gamma\right)<\beta+1 .
$$

This contradicts the continuity of $f_{1}$ at $\sup \left\{x_{n}\right\}=\sup \left\{\alpha_{n}\right\}$.

Claim. 3. The mapping $f_{1}$ is bounded on $L \times\{\gamma\}$.

Proof. By Claim 2 there is some $a \in L$ such that $f_{1}(x, \gamma) \leqslant \beta+2$ for all $x \geqslant a$. Since $[0, a] \times\{\gamma\}$ is compact it follows that $f_{1}$ is bounded on $L \times\{\gamma\}$.

Now let $b$ be an element of $L$ such that $b=\inf \left\{\gamma \in L \mid f_{1}\right.$ is bounded on $L \times\{\gamma\}\}$.

Claim 4. The mapping $f_{1}$ is bounded on $L \times\{b\}$.

Proof. Let $\left\{b_{n}\right\}$ be a monotonically decreasing sequence converging to $b$. For each $n$, let $m_{n}$ be an upper bound for $f_{1}$ on $L \times\left\{b_{n}\right\}$. Then $\sup \left\{m_{n} \mid n \in \omega_{0}\right\}$ is an upper bound for $f_{1}$ on $L \times\left\{b_{n}\right\}$.

Note that $b>0$ since $f$ is the identity on $L \times\{0\}$.

We now obtain our final contradiction.

Claim 5. The mapping $f_{1}$ is unbounded on $L \times\{b\}$.

Proof. Let $\lambda \in \omega_{1}$ be arbitrary. Let $\left\{b_{n}\right\}$ be a monotonically increasing sequence converging to $b$. For each $n, f_{1}$ is unbounded on $L \times\left\{b_{n}\right\}$. Hence $\left\{x \in L \mid f_{1}\left(x, b_{n}\right)\right.$ $\geqslant \lambda\}$ is a closed and unbounded subset of $L$. Thus, by the continuity of $f_{1}$, $\left\{\alpha \in \omega_{1} \mid f_{1}\left(\alpha, b_{n}\right) \geqslant \lambda\right\}$ is a closed and unbounded subset of $L$. Therefore the intersection

$$
\bigcap_{n \in \omega_{0}}\left\{\alpha \in \omega_{1} \mid f_{1}\left(\alpha, b_{n}\right) \geqslant \lambda\right\}
$$

is a closed and unbounded subset of $\omega_{1}$. The continuity of $f$ ensures that, for each $\alpha$ in this set, we have $f_{1}(\alpha, b) \geqslant \lambda$. But, since $\lambda$ was arbitrary, it follows that $f_{1}$ is unbounded on $L \times\{b\}$ as required. 
We remark that if we replace $L$ by $X=L-\{0\}$ and let $K=X \times\{1\} \cup\{1\} \times X$ in Theorem 3.6, then we obtain a nonmetric manifold without boundary which fails to have CIP.

Although it is easy to show that property $W$ (and, of course, property $W$ (strong)) is preserved by arbitrary products, an uncountable product of nondegenerate spaces will never be perfectly normal. Consequently, Ward's Lemma cannot be used to determine if such a product has CIP. The following theorem gives sufficient conditions for an infinite product of spaces to have CIP.

We shall adopt the notation that if $\left\{X_{\alpha} \mid \alpha \in A\right\}$ is a nonempty family of spaces and $B \subset A$, then $\pi_{B}: \Pi\left\{X_{\alpha} \mid \alpha \in A\right\} \rightarrow \prod\left\{X_{\alpha} \mid \alpha \in B\right\}$ denotes the surjective mapping between the two product spaces defined by $\pi_{B}(x)=x \mid B$. As usual, for any ordinal $\beta$ we consider $\beta=\{\alpha \mid \alpha \in B\}=\{\alpha \mid \alpha<\beta\}$.

3.7. THEOREM. Let $\lambda$ be an infinite cardinal. For each $\alpha \in \lambda$, let $Y_{\alpha}$ be a topological space with a basis $\{B(\alpha, \gamma) \mid \gamma \in \lambda\}$. Furthermore, suppose that for each pair of finite subsets $F$ and $G$ of $\lambda$ such that $F=\left\{\alpha_{1}, \ldots, \alpha_{n}\right\}, G=\left\{\gamma_{1}, \ldots, \gamma_{n}\right\}$ and each nonzero $\beta \in \lambda$, there is a mapping $h: Y_{\alpha_{1}} \times \cdots \times Y_{\alpha_{n}} \times Y_{\beta} \rightarrow Y_{\beta}$ such that, for any $x \in Y_{\alpha_{1}}$ $\times \cdots \times Y_{\alpha_{n}} \times Y_{\beta}, h(x)=\pi_{\{\beta\}}(x)$ iff $\pi_{F}(x) \notin B\left(\alpha_{1}, \gamma_{1}\right) \times \cdots \times B\left(\alpha_{n}, \gamma_{n}\right)$. Then $\Pi\left\{Y_{\alpha} \mid \alpha \in \lambda\right\}$ has $C I P$.

Proof. Let $\left\{\left(F_{\beta}, G_{\beta}\right) \mid \beta \in \lambda\right\}$ enumerate $\{(F, G) \mid F$ and $G$ are finite subsets of $\lambda\}$ in such a way that $F_{\beta} \subset \beta$ for all $\beta \in \lambda$.

Let $K$ be a closed subset of $Y=\Pi\left\{Y_{\alpha} \mid \alpha \in \lambda\right\}$. Construct a mapping $f: Y \rightarrow Y$, by constructing, for each $1 \leqslant \beta \leqslant \lambda$, a mapping $f_{\beta}$ : $\left\{Y_{\alpha} \mid \alpha \in \beta\right\} \rightarrow \Pi\left\{Y_{\alpha} \mid \alpha \in \beta\right\}$ such that:

(i) $\pi_{\mu} f_{\beta}(x)=f_{\mu} \pi_{\mu}(x)$ for all $\mu<\beta$ and all $x \in \Pi\left\{Y_{\alpha} \mid \alpha \in \beta\right\}$, and

(ii) $f_{\beta}(x)=x$ for all $x \in \pi_{\beta}(K)$.

At stage 1 let $f_{1}: Y_{0} \rightarrow Y_{0}$ be the identity.

At stage $\beta+1$ there are two cases; in both we assume a mapping $f_{\beta}$ has been constructed to satisfy (i) and (ii).

Case 1. Here $F_{\beta}=\left\{\alpha_{1}, \ldots, \alpha_{n}\right\}, G_{\beta}=\left\{\gamma_{1}, \ldots, \gamma_{n}\right\}$ and $B\left(\alpha_{1}, \gamma_{1}\right) \times \cdots \times$ $B\left(\alpha_{n}, \gamma_{n}\right) \cap \pi_{F_{\beta}}(K)=\varnothing$. In this case we use the mapping

$$
h: Y_{\alpha_{1}} \times \cdots \times Y_{\alpha_{n}} \times Y_{\beta} \rightarrow Y_{\beta}
$$

given by hypothesis and we let $f_{\beta+1}$ be defined so that, if $x \in \Pi\left\{Y_{\alpha} \mid \alpha<\beta+1\right\}$,

(iii) $\pi_{\beta} f_{\beta+1}(x)=f_{\beta} \pi_{\beta}(x)$,

(iv) $\pi_{\{\beta\}} f_{\beta+1}(x)=h \pi_{F_{\beta} \cup\{\beta\}}(x)$.

Case 2. If Case 1 is not in effect, define $\pi_{\beta} f_{\beta+1}(x)$ as in (iii), but make

(v) $\pi_{\{\beta\}} f_{\beta+1}(x)=\pi_{\{\beta\}}(x)$.

Since the projection onto each factor is continuous, $f_{\beta+1}$ is continuous. Furthermore, (i) is satisfied by virtue of (iii), and (ii) by virtue of (iv) and (v).

At the limit stage $\delta \leqslant \lambda$, assume $f_{\beta}$ is a mapping satisfying (i) and (ii) for each $\beta<\delta$. For $x \in \prod\left\{Y_{\alpha} \mid \alpha<\delta\right\}$, define $f_{\delta}$ so that

(vi) $\pi_{\{\beta\}} f_{\delta}(x)=\pi_{\{\beta\}} f_{\beta+1} \pi_{\beta+1}(x)$.

Since (i) holds for all $\beta<\delta$, this definition is unambiguous and since each projection is continuous, so is $f_{\delta}$. 
This completes the construction; we let $f=f_{\lambda}$. It remains to show that if $y \notin K$, then $f(y) \neq y$.

If $y \notin K$, there is an elementary open neighborhood of $y$ disjoint from $K$. Thus there exists $F=\left\{\alpha_{1}, \ldots, \alpha_{n}\right\} \subset \lambda$ and $G=\left\{\gamma_{1}, \ldots, \gamma_{n}\right\} \subset \lambda$ such that $\pi_{F}(y) \in$ $B\left(\alpha_{1}, \gamma_{1}\right) \times \cdots \times B\left(\alpha_{n}, \gamma_{n}\right) \subset \pi_{F}(Y-K)$. Let $\beta \in \lambda$ be such that $F=F_{\beta}$ and $G=G_{\beta}$. Then the construction of $f_{\beta+1}$ is such that $\pi_{\{\beta\}} f_{\beta+1} \pi_{\beta+1}(y)=h \dot{\pi}_{F \cup\{\beta\}}(y)$ where $h$ is as in (iv). Hence $\pi_{\{\beta\}} f_{\beta+1} \pi_{\beta+1}(y) \cdot \neq \pi_{\{\beta\}}(y)$ and so $\pi_{\{\beta\}} f(y) \neq \pi_{\{\beta\}}(y)$. Thus $f(y) \neq y$ as required.

3.8. Lemma. Suppose for each $\alpha \in \lambda, Y_{\alpha}$ is a locally metrizable space such that there is a deformation $H_{\alpha}: Y_{\alpha} \times I \rightarrow Y_{\alpha}$ such that $H_{\alpha}(y, t)=y$ iff $t=0$ (i.e., each $Y_{\alpha}$ has property $W$ (strong)). Then $\left\{Y_{\alpha} \mid \alpha \in \lambda\right\}$ satisfies the hypothesis of Theorem 3.7 regarding $h$.

Proof. Without loss of generality we assume that each of the closed sets $\mathrm{Cl}(B(\alpha, \gamma))$ is metrizable. Define $h: Y_{\alpha_{1}} \times \cdots \times Y_{\alpha_{n}} \times Y_{\beta} \rightarrow Y_{\beta}$ by

$$
h(x)=H_{\beta}\left(\pi_{\{\beta\}}(x), \frac{d(x)}{1+d(x)}\right)
$$

where $d(x)=\operatorname{dist}\left(\pi_{F}(x), Y_{\alpha_{1}} \times \cdots \times Y_{\alpha_{n}}-B\left(\alpha_{1}, \gamma_{1}\right) \times \cdots \times B\left(\alpha_{n}, \gamma_{n}\right)\right)$ if $\pi_{F}(x)$ $\in B\left(\alpha_{1}, \gamma_{1}\right) \times \cdots \times B\left(\alpha_{n}, \gamma_{n}\right)$, and $d(x)=0$ otherwise.

We note that nonmetric manifolds with property $W$ (strong) satisfy Lemma 3.8. Thus, for instance, although it was reasonably difficult to establish that $L \times S^{1}$ has CIP, it follows immediately from Theorem 3.7 and Lemma 3.8 that any uncountable self-product of $L \times S^{1}$ has CIP.

The next result follows from Ward's Lemma when $\lambda$ is countable, and is an immediate consequence of Theorem 3.7 and Lemma 3.8 when $\lambda$ is any infinite cardinal.

3.9. THEOREM. Let $\left\{Y_{\alpha} \mid \alpha \in \lambda\right\}$ be a nonempty family of separable metric spaces having property $W$ (strong). Then the product space $\Pi\left\{Y_{\alpha} \mid \alpha \in \lambda\right\}$ has CIP.

We remark that since there is a substantial class of separable metric spaces having property $W$ (strong), Theorem 3.9 yields a substantial class of nonmetric spaces and groups having CIP in the case when $\lambda$ is not countable. For instance, it follows that an uncountable product of circles or real lines must have CIP.

3.10. LEMMA. Suppose for each $\alpha \in \lambda, Y_{\alpha}$ is a nondegenerate 0-dimensional space. Then $\left\{Y_{\alpha} \mid \alpha \in \lambda\right\}$ satisfies the hypothesis of Theorem 3.7 regarding $h$.

Proof. First without loss of generality we may assume that all the sets $B(\alpha, \gamma)$ are clopen. Then $h: Y_{\alpha_{1}} \times \cdots \times Y_{\alpha_{n}} \times Y_{\beta} \rightarrow Y_{\beta}$ is defined as follows: Let $U$ be a clopen set in $Y_{\beta}$ such that $a \in U, b \notin U$, and set

$$
h\left(\pi_{F}(x), \pi_{\{\beta\}}(x)\right)=\left\{\begin{array}{l}
\pi_{\{\beta\}}(x) \text { if } \pi_{F}(x) \notin B\left(\alpha_{1}, \gamma_{1}\right) \times \cdots \times B\left(\alpha_{n}, \gamma_{n}\right), \\
a \quad \text { if } \pi_{F}(x) \in B\left(\alpha_{1}, \gamma_{1}\right) \times \cdots \times B\left(\alpha_{n}, \gamma_{n}\right), \pi_{\{\beta\}}(x) \notin U, \\
b \quad \text { if } \pi_{F}(x) \in B\left(\alpha_{1}, \gamma_{1}\right) \times \cdots \times B\left(\alpha_{n}, \gamma_{n}\right), \pi_{\{\beta\}}(x) \in U .
\end{array}\right.
$$


The following immediate corollary to Theorem 3.7 and Lemma 3.10 yields a class of totally disconnected groups having CIP.

3.11. THEOREM. Let $\left\{Y_{\alpha} \mid \alpha \in \lambda\right\}$ be a nonempty family of discrete spaces, each of which has at most a countable number of elements. Then the product space $\Pi\left\{Y_{\alpha} \mid \alpha \in \lambda\right\}$ has CIP.

In the following theorem we show that in order for a certain type of product space to have CIP, it is both necessary and sufficient that the product space be perfectly normal.

3.12. THEOREM. Let $M$ be a connected metric space with property $W$ (strong) and let $Y$ be a totally disconnected normal space. Then $M \times Y$ has CIP iff $Y$ is perfectly normal.

Proof. Sufficiency. Let $Y$ be a perfectly normal space. Then $M \times Y$ is a perfectly normal space $[4,4.5 .16(\mathrm{~b})$, p. 367] which has property $W$ (strong), and therefore has CIP by Ward's Lemma.

Necessity. Suppose $M \times Y$ has CIP and $Y$ is not perfectly normal. Since $Y$ is a normal space it follows that there is a closed subset $K$ of $Y$ which is not a $G_{\delta}$-set. Let $p \in M$ and let $A$ be the closed subset of $M \times Y$ defined by

$$
A=\{(x, y) \in M \times Y \mid x=p \text { or } y \in K\} .
$$

Then there is a mapping $f: M \times Y \rightarrow M \times Y$ whose fixed point set is $A$. Since $M$ is connected, $Y$ is totally disconnected and $f(p, y)=(p, y)$ for each $y \in Y$, it follows that $f(M \times\{y\}) \subset M \times\{y\}$ for each $y \in Y$.

Choose a point $q \neq p$ in $M$ and let $\delta=\rho(p, q)$ where $\rho$ denotes the metric on $M$. Let $f_{1}=\pi f$ where $\pi: M \times Y \rightarrow M$ denotes the natural projection mapping and, for $n=1,2, \ldots$, define

$$
U_{n}=\left\{y \in Y \mid \rho\left(q, f_{1}(q, y)\right)>\delta / n\right\} .
$$

Then $Y-K=\bigcup_{n=1}^{\infty} U_{n}$. Moreover, since $Y-K$ is not an $F_{\mathrm{o}}$-set, it follows that $Y-K \neq \bigcup_{n=1}^{\infty} \operatorname{Cl}\left(U_{n}\right)$. Thus, for some $U_{n}$, there is a $z$ such that $z \in K \cap \operatorname{Cl}\left(U_{n}\right)$. Hence $f(q, z)=(q, z)$ and $f_{1}(q, z)=q$. But $\rho\left(q, f_{1}(q, y)\right) \geqslant \delta / n$ for all $y \in \operatorname{Cl}\left(U_{n}\right)$. This contradiction shows that $Y$ must be perfectly normal as required.

We have previously mentioned that any metric group which is either locally compact or contains an arc must have CIP [9, p. 1028]. It seems appropriate to ask if an analogous situation exists for nonmetric groups. Indeed, in Question 3 of [12, p. 422], H. Schirmer asks if there exists a nonmetrizable group which fails to have CIP. In the following result we show that products of compact groups need not preserve CIP. In fact, we show that if $S^{1}$ denotes the circle group and $G$ is a compact group having CIP, then $S^{1} \times G$ need not have CIP.

3.13. TheOREM. Let $\lambda$ be an uncountable cardinal and let $\left(Z_{2}\right)^{\lambda}$ denote the self-product of $\lambda$ copies of the two-point discrete group $Z_{2}=\{0,1\}$. Then $S^{1} \times\left(Z_{2}\right)^{\lambda}$ does not have CIP. 
Proof. The compact totally disconnected group $\left(Z_{2}\right)^{\lambda}$ has CIP by Theorem 3.11 . Moreover, since $\left(Z_{2}\right)^{\lambda}$ is not perfectly normal, it follows from Theorem 3.12 that $S^{1} \times\left(Z_{2}\right)^{\lambda}$ does not have CIP.

In the next result we use the concept of CIP to give a characterization of the compact 0 -dimensional spaces which are metrizable.

3.14. THEOREM. Let $M$ be a connected metric space with property $W$ (strong) and let $X$ be a compact 0-dimensional space. Then $M \times X \times X$ has CIP iff $X$ is metrizable.

Proof. Sufficiency. If $X$ is metrizable, then $M \times X \times X$ has CIP by Ward's Lemma.

Necessity. Suppose $M \times X \times X$ has CIP. Since $X \times X$ is a compact totally disconnected space, it follows from Theorem 3.12 that $X \times X$ is perfectly normal. Thus the diagonal is a $G_{\delta}$-set in $X \times X$, and therefore $X$ is metrizable [4, 4.2B, p. 330].

In order to show that the Stone-Čech compactification of the integers does not have CIP, we prove the following preliminary lemma.

3.15. Lemma. Let $f: N \rightarrow N$ be a self-mapping of the positive integers $N$. Then either

(i) there is an infinite subset $A$ of $N$ such that for all $x \in A, f(x)=x$, or

(ii) there is an infinite subset $A$ of $N$ such that for all $x \in A, f(x) \notin A$.

Proof. There are two cases to consider.

Case 1. Suppose there is some $y \in N$ such that $f^{-1}(y)$ is infinite. Then $A=f^{-1}(y)$ $-\{y\}$ satisfies (ii).

Case 2. Suppose for each $y \in N, f^{-1}(y)$ is finite. Let $x_{0} \in N$ and, for each integer $n \geqslant 1$, let $x_{n}$ be a positive integer such that

$$
x_{n} \notin f^{-1}\left(\left\{x_{0}, \ldots, x_{n-1}\right\}\right) \cup\left\{f\left(x_{0}\right), \ldots, f\left(x_{n-1}\right)\right\} .
$$

Then either $\left\{x_{n} \mid f\left(x_{n}\right)=x_{n}\right\}$ is infinite and satisfies (i), or $\left\{x_{n} \mid f\left(x_{n}\right) \neq x_{n}\right\}$ is infinite and satisfies (ii).

3.16. THEOREM. The Stone-Čech compactification $\beta N$ of $N$ does not have CIP, but the self-product $(\beta N)^{\mathbf{c}}$, where $\mathbf{c}$ denotes the cardinal of the continuum, does have CIP.

Proof. It follows from Theorem 3.7 and Lemma 3.10 that $(\beta N)^{\mathbf{c}}$ has CIP.

Now suppose $f: \beta N \rightarrow \beta N$ is a mapping whose fixed point set is $\beta N-N$. By Lemma 3.15 there is an infinite subset $A$ of $N$ such that

$$
f(A) \subset(N-A) \cup((\beta N-N) \cap f(A)) .
$$

Identify $x \in N$ with the ultrafilter fixed at $x$. Let

$$
U_{A}=\{\phi \mid \phi \text { is an ultrafilter and } A \in \phi\}
$$

and similarly define $U_{N-A}$. Then 


$$
\begin{aligned}
U_{A} \cap f\left(U_{A}\right) & \subset U_{A} \cap \mathrm{Cl}(f(A)) \\
& \subset U_{A} \cap(\mathrm{Cl}(N-A) \cup \mathrm{Cl}((\beta N-N) \cap f(A))) \\
& =U_{A} \cap\left(U_{N-A} \cup \mathrm{Cl}((\beta N-N) \cap f(A))\right) \\
& =U_{A} \cap \mathrm{Cl}((\beta N-N) \cap f(A)) \\
& \subsetneq U_{A} \cap(\beta N-N) .
\end{aligned}
$$

The last inclusion is proper since an open subset of $\beta N-N$ is not the closure of a countable subset of itself. Consequently,

$$
f\left(U_{A} \cap(\beta N-N)\right) \neq U_{A} \cap(\beta N-N)
$$

and this contradiction shows that $\beta N$ does not have CIP as required.

To facilitate the following discussion, we shall call $Y$ a (multiplicative) CIPcompanion of $X$ if $X \times Y$ has CIP and say that $Y$ is an absolute CIP-companion for a class $Q$ of spaces if $Y$ is a CIP-companion for each $X \in Q$. Some of the examples in this paper are those of spaces which possess CIP and which are not CIP-companions of themselves (see 2.4, 3.6 and 4.3). From Ward's Lemma it follows that $S^{1}$ is an absolute CIP-companion for the class of perfectly normal spaces. However, results in this section show that $S^{1}$ fails to be an absolute CIP-companion for the class of compact spaces. Indeed, we have seen that $L^{*} \times S^{1}$ fails to have CIP and $\left(Z_{2}\right)^{\mathbf{c}} \times S^{1}$, $\beta N \times S^{1},(\beta N)^{\mathbf{c}} \times S^{1}$ all fail to have CIP by virtue of Theorem 3.12. For certain spaces one can clearly choose a suitable self-product of $S^{1}$ in order to obtain a CIP-companion. For instance, although $\left(Z_{2}\right)^{\mathbf{c}} \times S^{1}$ fails to have CIP, $\left(S^{1}\right)^{\mathbf{c}}$ is a CIP-companion of $\left(Z_{2}\right)^{\mathbf{c}}$ since $\left(Z_{2}\right)^{\mathbf{c}} \times\left(S^{1}\right)^{\mathbf{c}}$ is homeomorphic to $\left(Z_{2} \times S^{1}\right)^{\mathbf{c}}$ which has CIP by Theorem 3.9. The next theorem asserts that every completely regular space has a CIP-companion which is a self-product of $S^{1}$.

3.17. THEOREM. Let $Y$ be a completely regular space. Then there is a cardinal $\lambda$ such that $Y \times\left(S^{1}\right)^{\lambda}$ has $C I P$.

Proof. In the case where $Y$ is perfectly normal we have seen that $\lambda=1$ suffices. Thus assume that $Y$ is a completely regular space which is not perfectly normal. In this case we can choose an infinite cardinal $\lambda$ and a basis $\{B(0, \gamma) \mid \gamma<\lambda\}$ of cozero sets for $Y$. Let $\left\{Y_{\alpha} \mid \alpha \in \lambda\right\}$ denote the family of spaces such that $Y_{0}=Y, Y_{\alpha}=S^{1}$ if $\alpha \neq 0$, and let $\beta, F, G$ be as in 3.7. Now we complete the proof by defining a mapping $h$ which satisfies the hypothesis of Theorem 3.7. There are two cases to consider.

Case 1. If $0 \notin F$, then we let $h$ be defined as in the proof of Lemma 3.8.

Case 2. If $0 \in F$, let $F=\left\{0, \alpha_{1}, \ldots, \alpha_{n}\right\}$ and $G=\left\{\gamma_{0}, \gamma_{1}, \ldots, \gamma_{n}\right\}$. Let $\rho$ be a metric on $Y_{\alpha_{1}} \times \cdots \times Y_{\alpha_{n}}$ which is bounded by 1 and let $f: Y_{0} \rightarrow I$ be a mapping such that $B\left(0, \gamma_{0}\right)=f^{-1}((0,1])$. For $x \in Y_{0} \times Y_{\alpha_{1}} \times \cdots \times Y_{\alpha_{n}} \times Y_{\beta}$, let $e^{i \theta}=$ $\pi_{\{\beta\}}(x)$ and define

$$
d(x)=\rho\left(\pi_{F-\{0\}}(x), Y_{\alpha_{1}} \times \cdots \times Y_{\alpha_{n}}-B\left(\alpha_{1}, \gamma_{1}\right) \times \cdots \times B\left(\alpha_{n}, \gamma_{n}\right)\right) .
$$

We define the mapping $h: Y_{0} \times Y_{\alpha_{1}} \times \cdots \times Y_{\alpha_{n}} \times Y_{\beta} \rightarrow Y_{\beta}$ by

$$
h(x)=e^{i\left(\theta+d(x) f\left(\pi_{\{0\}}(x)\right)\right)} \quad \text { if } x \in Y_{0} \times Y_{\alpha_{1}} \times \cdots \times Y_{\alpha_{n}} \times Y_{\beta} .
$$


It follows that $h(x)=\pi_{\{\beta\}}(x)$ iff $\pi_{F}(x) \notin B\left(0, \gamma_{0}\right) \times B\left(\alpha_{1}, \gamma_{1}\right) \times \cdots \times B\left(\alpha_{n}, \gamma_{n}\right)$ and this completes the proof.

3.18. REMARK. In the proof of Theorem 3.17 , the product $\left(S^{1}\right)^{\lambda}$ could be replaced by a product of $\lambda$ metric spaces each of which has property $W$ (strong) and weight $\leqslant \lambda$. In fact, using the notation of Lemma 3.8, the only adjustment required would be to replace $e^{i\left(\theta+d(x) f\left(\pi_{\{0\}}(x)\right)\right)}$ by $H_{\beta}\left(\pi_{\{\beta\}}(x), d(x) f\left(\pi_{\{0\}}(x)\right)\right)$.

The following result, whose proof relies on a basic lemma in the next section, shows that there is no absolute CIP-companion for the class of completely regular spaces.

3.19. THEOREM. For each completely regular space $C$ there is a completely regular space $X$ such that $X \times C$ does not have $C I P$.

Proof. Suppose $C$ is a completely regular space of cardinality $\alpha$. Let $\lambda=2^{\alpha}$ (the cardinality of all subsets of $C$ ). Then the self-product $\left(Z_{2}\right)^{\lambda}$ has a dense subset $D$ of cardinality $\alpha[4,2.3 .15$, p. 111] and a discrete subspace $E$ of cardinality $\lambda[4,6.2 .16$, p. 447]. If $X=D \cup E$, then $X \times C$ is a completely regular space with a dense subset of cardinality $\alpha$ and a discrete subspace of cardinality $\lambda$. Moreover, the cardinality of the set of self-mappings of $X \times C$ is $\lambda$. Since $2^{\lambda}>\lambda$, it follows from Lemma 4.1 that $X \times C$ does not have CIP.

4. Independence results. In this section we further discuss the complete invariance property in the context of nonmetrizable spaces. To begin with, we consider a topological space formed by making adjustments to the topology of a subset of the closed upper half-plane $R_{+}^{2}$. We show that the presence of CIP in this space is neither provable nor refutable with the usual axioms of set theory. The following lemma is useful in this context.

4.1. Lemma. Suppose $X$ is a space with a discrete subset of cardinality $\lambda$ and $2^{\lambda}$ is greater than the cardinality of the collection of all self-mappings of $X$. Then $X$ does not have CIP.

Proof. Let $D$ be the discrete subset of $X$ of cardinality $\lambda$. Since distinct subsets of $D$ have distinct closures in $X$, there are at least $2^{\lambda}$ distinct closed subsets of $X$. Thus, for $X$ to have CIP, at least $2^{\lambda}$ self-mappings of $X$ are required.

We now consider the so-called bubble space $B$ or Niemytzki plane (see [4, p. 39]). Let $A$ be a subset of the real numbers $R$ of cardinality $\aleph_{1}$, the smallest uncountable cardinal. The underlying set of $B$ is the union of the open upper half-plane $\{(x, y) \mid x, y \in R, y>0\}$ and the set $\{(x, 0) \mid x \in A\}$. The topology on $B$ is generated by the usual Euclidean topology on the open upper half-plane and sets of the form $\left\{(x, y) \mid(x-a)^{2}+(y-r)^{2}<r^{2}\right\} \cup\{(a, 0)\}$ where $a \in A$ and $r>0$. Your mental picture of a basis for this space may be a collection of bubbles floating up from the $x$-axis.

Does the bubble space $B$ have CIP?

4.2. THEOREM. The statement " $B$ has $C I P$ " is neither provable nor refutable with the usual axioms of set theory. Furthermore: 
(i) $\mathrm{CH}$ implies that $B$ does not have $C I P$, and

(ii) MA plus $\neg \mathrm{CH}$ implies that $B$ does have $C I P$.

Proof. We first explain the second sentence. $\mathrm{CH}$ denotes the continuum hypothesis, $2^{\aleph_{0}}=\aleph_{1}$, which was proven by K. Gödel to be relatively consistent with the usual axioms of set theory (i.e., ZFC, the Zermelo-Fraenkel axioms including the axiom of choice). That is, if $\mathrm{ZFC}$ is consistent, so is $\mathrm{ZFC}$ together with $\mathrm{CH}$. Therefore, we can conclude that, barring an inconsistency within $\mathrm{ZFC}$, nothing provable from $\mathrm{ZFC}$ plus $\mathrm{CH}$ can be refuted by $\mathrm{ZFC}$ alone.

MA plus $\neg \mathrm{CH}$ denotes Martin's Axiom together with the negation of the continuum hypothesis. This combination was proven by R. Solovay and S. Tennenbaum to be relatively consistent with ZFC. Martin's Axiom is usually stated in a form dealing with partial orders, but it is equivalent to the following statement.

"No compact Hausdorff topological space, in which every pairwise disjoint collection of open subsets is countable, is the union of fewer than $2^{\aleph_{0}}$ nowhere dense subsets."

For our purposes, we need the fact that MA plus $\neg \mathrm{CH}$ implies that $B$ is a perfectly normal space (see [7 or 13]). For more information concerning the above discussion, the reader may consult appropriate articles in [1 or 8].

We now turn to the proof of (i). Every self-mapping of $B$ is, of course, determined by its restriction to a dense subset of $B$. Therefore, since $B$ is a separable space of cardinality $2^{\aleph_{0}}=\mathbf{c}$, there can be at most $\mathrm{c}$ self-mappings of $B$. Now the set $\{(a, 0) \mid a \in A\}$ is a discrete subset of $B$ of cardinality $\boldsymbol{\aleph}_{1}$. Since $2^{\mathbf{c}}>\mathbf{c}, \mathrm{CH}$ and Lemma 4.1 combine to ensure that $B$ does not have CIP.

On the other hand, to prove (ii) we use Martin's Axiom and Ward's Lemma. In this case, the space $B$ is perfectly normal and it suffices to show that $B$ has property $W$ (strong). But this is witnessed by the deformation $H: B \times I \rightarrow B$ defined by

$$
H((x, y), t)=(x, y+t) \quad \text { if }(x, y) \in B, t \in I .
$$

Thus the proof is complete.

We now turn out attention to the right half-open interval space $K$, sometimes called the Sorgenfrey line (see [4, p. 39]). The underlying set of $K$ is the set of all real numbers $R$, and a basis for the topology of $K$ is the family of all sets of the form

$$
[a, b)=\{x \mid a \leqslant x<b\} \quad \text { where } a, b \in R .
$$

The relationship between CIP and powers (i.e., self-products) of $K$ is rather intriguing.

4.3. TheOREM. Let $K$ be the Sorgenfrey line. Then:

(i) $K$ has CIP.

(ii) $K^{2}$ does not have CIP.

(iii) For each integer $n \geqslant 2, K^{n}$ does not have CIP.

(iv) The countably infinite power $K^{\boldsymbol{\aleph}_{1}}$ does not have CIP.

(v) For each cardinal $\lambda \geqslant \mathbf{c}, K^{\lambda}$ does have CIP. 
Proof. In order to prove (i), we note that every open subset of $K$ can be realized as a countable disjoint union of half-open intervals of the form $[a, b)$, and that $x \rightarrow(x+b) / 2$ determines a fixed point free homeomorphism of $[a, b)$ into itself. It follows that every nonempty closed subset $A$ of $K$ is the fixed point set of a self-mapping of $K$ which maps $K-A$ homeomorphically into itself.

To prove (i), (iii) and (iv), we use Lemma 4.1. The space $K^{2}$ is separable [4, 2.3.12, p. 111] and has cardinality c. Thus there are only $\mathbf{c}^{\boldsymbol{\aleph}_{0}}=\mathbf{c}$ self-mappings of $K^{2}$. On the other hand, $\{(x,-x) \mid x \in K\}$ is a discrete subset of $K^{2}$ with cardinality c. Since $2^{\mathbf{c}}>\mathbf{c}$, the proof of (ii) follows from Lemma 4.1, and the same proof is valid for (iii) and (iv).

The proof of (v) follows from Theorem 3.7 and Lemma 3.10 since $K$ is a 0-dimensional space with a basis of cardinality c $[4,1.2 .2$, p. 39].

We have not completely determined the relationship between CIP and all powers of $K$. In particular, we have yet to resolve the question: Does $K^{\aleph_{1}}$ have CIP? In fact, this is another independence result.

4.4. ThEOREM. Let $K$ be the Sorgenfrey line. Then:

(i) $\mathrm{CH}$ implies that $K^{\aleph_{1}}$ has $C I P$, and

(ii) MA plus $\neg \mathrm{CH}$ implies that $K^{\aleph_{1}}$ does not have CIP.

Proof. First suppose $\mathrm{CH}$ holds. Then $\boldsymbol{\aleph}_{1}=2^{\aleph_{0}}=\mathrm{c}$ and therefore $K^{\aleph_{1}}$ has CIP by Theorem 4.3(v).

Now suppose MA plus $\neg \mathrm{CH}$ holds. Then $2^{\aleph_{1}}=2^{\aleph_{0}}=\mathbf{c}$ and hence $2^{\mathbf{c}}>2^{\aleph_{1}}$. (For a reference consult the article by M. E. Rudin in [1].) Therefore, as in the proof of Theorem 4.3(ii), $K^{\aleph_{1}}$ has a discrete subset of cardinality c, and has only $2^{\aleph_{1}}$ self-mappings since it is separable [4, 2.3.15, p. 111]. It follows from Lemma 4.1 that $K^{\aleph_{1}}$ does not have CIP.

Ward's Lemma guarantees that countable products of metric spaces having property $W$ (strong) will have CIP. However, perfect normality is not a finitely multiplicative property. Consequently, no such assurance can be given for products of perfectly normal spaces having property $W$ (strong), as is shown in the next example.

4.5. EXAmple. The space $K \times S^{1}$ is a perfectly normal space $[4$, p. 68 and 4.5.16(b), p. 367] which has property $W$ (strong) and, hence, CIP. However, its square $\left(K \times S^{1}\right)^{2}$ is homeomorphic to $K \times K \times S^{1} \times S^{1}$. Clearly, the latter is a separable space of cardinality $\mathbf{c}$ which contains a discrete subspace of cardinality $\mathbf{c}$. Thus, once again, Lemma 4.1 can be applied to ensure that the product $\left(K \times S^{1}\right)^{2}$ does not have CIP.

\section{REFERENCES}

1. J. Barwise (ed.), Handbook of mathematical logic, North-Holland, Amsterdam, 1977.

2. H. Cook, Continua which admit only the identity mapping onto nondegenerate subcontinua, Fund. Math. 60 (1967), 241-249.

3. K. J. Devlin, Fundamentals of contemporary set theory, Springer-Verlag, Berlin, 1979.

4. R. Engelking, General topology, Monogr. Mat., Vol. 60, PWN, Warsaw, 1977. 
5. Boju Jiang and H. Schirmer, Fixed point sets of continuous selfmaps on polyhedra, Fixed Point Theory Proc. (Sherbrooke, 1980), Lecture Notes in Math., vol. 866, Springer-Verlag, Berlin, 1981, pp. 171-177.

6. F. B. Jones, Connected and disconnected plane sets and the functional equation $f(x)+f(y)=f(x+y)$, Bull. Amer. Math. Soc. 48 (1942), 115-120.

7. I. Juhász and W. Weiss, Martin's axiom and normality, Gen. Topology Appl. 9 (1978), 263-274.

8. K. Kunen and J. Vaughan (eds.), Handbook of set-theoretic topology, North-Holland, Amsterdam (to appear).

9. J. R. Martin and S. B. Nadler, Jr., Examples and questions in the theory of fixed point sets, Canad. J. Math. 31 (1979), 1017-1032.

10. J. R. Martin and E. D. Tymchatyn, Fixed point sets of 1-dimensional Peano continua, Pacific J. Math. 89 (1980), 147-149.

11. J. R. Martin, L. G. Oversteegen and E. D. Tymchatyn, Fixed point sets of products and cones, Pacific J. Math. 101 (1982), 133-139.

12. H. Schirmer, Fixed point sets of continuous selfmaps, Fixed Point Theory Proc. (Sherbrooke, 1980), Lecture Notes in Math., vol. 866, Springer-Verlag, Berlin, 1981, pp. 417-428.

13. F. D. Tall, Set-theoretic consistency results and topological theorems concerning the normal Moore space conjecture and related problems, $\mathrm{Ph} . \mathrm{D}$. Thesis, University of Wisconsin, Madison, 1969.

14. L. E. Ward, Jr., Fixed point sets, Pacific J. Math. 47 (1973), 553-565.

Department of Mathematics, University of Saskatchewan, Saskatoon, SaSkatchewan, Canada S7N 0W0

Department of Mathematics, University of Toronto, Toronto, Ontario, Canada M5S 1A1 